\title{
Autonomic function testing in the COVID-19 pandemic: an American Autonomic Society position statement
}

\author{
Juan J. Figueroa ${ }^{1}$ - William P. Cheshire ${ }^{2}$. Victoria E. Claydon ${ }^{3} \cdot$ Lucy Norcliffe-Kaufmann $^{4} \cdot$ Amanda Peltier $^{5}$. \\ Wolfgang Singer ${ }^{6} \cdot$ Howard Snapper $^{7} \cdot$ Steven Vernino ${ }^{8} \cdot$ Satish R. Raj ${ }^{9,10}$ on behalf of The American Autonomic \\ Society
}

Received: 30 May 2020 / Accepted: 5 June 2020 / Published online: 11 June 2020

(c) Springer-Verlag GmbH Germany, part of Springer Nature 2020

\begin{abstract}
COVID-19 is a global pandemic that is wreaking havoc with the health and economy of much of human civilization. In this document from the American Autonomic Society, we identify the potential risks of exposure to patients, physicians, and allied healthcare staff. We provide guidance for conducting autonomic function testing safely in this environment.
\end{abstract}

\section{Statement of the problem}

The novel coronavirus (SARS-CoV-2) emerged in Wuhan, China, in late 2019. It quickly became a pandemic, significantly impacting the health and economy of the USA, many European countries, and the rest of the world [1,2]. There are hundreds of thousands of deaths related to COVID-19 (the disease caused by SARS-CoV-2) worldwide, with an estimated mortality rate ranging from $1 \%$ to $5 \%$ [2]. This healthcare crisis has imposed an unprecedented strain on society in general, and in particular it has challenged the ability of healthcare organizations to provide adequate care, including to patients without COVID-19. The purpose of this statement is to provide guidance to physicians, staff, and healthcare systems on the performance of autonomic function testing in these unusual times. There are no randomized trial data addressing these issues, so the thoughts here reflect

Satish R. Raj

satish.raj@ucalgary.ca

1 Department of Neurology, Medical College of Wisconsin, Milwaukee, WI, USA

2 Department of Neurology, Mayo Clinic, Jacksonville, FL, USA

3 Department of Biomedical Physiology and Kinesiology, Simon Fraser University, Burnaby, BC, Canada

4 Departments of Physiology and Neurosciences, New York University, New York, NY, USA

5 Autonomic Dysfunction Center, Department of Neurology, Vanderbilt University Medical Center, Nashville, TN, USA the opinions of leaders in autonomic disorders and testing with insights from experts in infection control.

\section{Potential risks of exposure to patients, physicians, and allied healthcare staff}

SARS-CoV-2 is a highly infectious virus associated with significant morbidity and mortality. Individuals may maintain high viral loads in the upper respiratory tract with significant potential for viral shedding and transmission even if asymptomatic [3, 4]. While primarily transmitted by droplets, airborne transmission is possible through aerosolization in the setting of intubation, endoscopy, or non-invasive positive pressure ventilation [5].

6 Department of Neurology, Mayo Clinic, Rochester, MN, USA

7 Cardiology Division, Wellstar Healthcare System, Atlanta, GA, USA

8 Department of Neurology and Neurotherapeutics, UT Southwestern Medical Center, Dallas, TX, USA

9 Autonomic Dysfunction Center, Division of Clinical Pharmacology, Department of Medicine, Vanderbilt University Medical Center, Nashville, TN, USA

10 Department of Cardiac Sciences, Libin Cardiovascular Institute, University of Calgary, GAC70 HRIC Building, 3280 Hospital Drive NW, Calgary, AB T2N 4Z6, Canada 


\section{Should the autonomic testing be performed or deferred?}

The COVID-19 pandemic has affected different countries, and different areas of individual countries, variably. In areas with a high case load, hospital procedures have sometimes been restricted to those that would be immediately life-saving or preventing potential harm if postponed. Autonomic testing would typically be deferred in those settings. As the pandemic resolves, or in less affected areas, autonomic testing could be resumed as long as it is felt to provide important clinical information. Virtual telehealth "visits" and patient self-assessment of orthostatic vital signs could be used to help guide the urgency of testing during these times.

As with any procedure, autonomic function testing should be limited to circumstances in which the information will guide clinical care.

\section{Screening of patient prior to autonomic testing procedure}

It is important that there be a high degree of suspicion for COVID-19 in every patient. A thorough travel history to current or emerging "hot spot" areas and assessment of contact with individuals/family members who were sick or received hospitalization is mandatory screening prior to autonomic testing. Patients with fever, cough, shortness of breath, anosmia, or any other new upper respiratory symptoms deserve special attention and should not undergo clinical autonomic testing until local testing protocols "clear them" of COVID19 infectivity. If exceptions are needed for research studies, institutional review board approval and a more stringent level of personal protective equipment as determined by the hospital infection control team are required.

\section{Autonomic function tests}

Autonomic function tests consist of a battery of testing involving continuous beat-to-beat blood pressure monitoring and electrocardiographic monitoring during head-up tilt, a series of breathing maneuvers, Valsalva maneuvers, and quantitative sudomotor testing [6]. Valid test results require a relaxed environment where the patient feels at ease, a condition that may be more difficult to achieve if the patient perceives a risk of becoming infected during the visit.

Visits should be scheduled to reduce patient volume in common waiting areas and patients should be advised, where possible, to bring only one additional support person or caregiver (if allowed by institutional policy). Where possible, to limit the face-to-face in office contact time, ancillary information to the testing may be collected with hybrid visits. For example, a brief focused clinical history, questionnaires, and medication reconciliation can be performed in a prior telemedicine virtual visit so that the in-office time is restricted to only autonomic testing procedures. Modifications to patient waiting areas and staff rooms to ensure proper social distancing may be necessary.

Hand sanitizer should be available on check-in. Patients should have undergone the institution-approved clinical COVID screening prior to entering the lab. The number of staff present in the room during autonomic testing should be kept to a minimum.

Patients should wear masks and not remove them while sitting in waiting areas, and when being taken to the examination or testing room. Masks should be worn during all parts of autonomic testing as appropriate and where the mask does not interfere with performance or interpretation of the test. Patients should wear masks during sudomotor testing without affecting the test results. Patients should wear masks during the breathing tests and the head-up tilt table test, if they are tolerated; if there is concern that the masks alter the breathing and secondarily the heart rate and blood pressure responses, it would be appropriate to remove the mask for those tests. During the head-up tilt table test, nausea and dyspnea can occur; if there is concern that masking may worsen nausea or dyspnea or otherwise influence the test results, it would be appropriate to remove the mask during tilt.

A proper Valsalva maneuver test would not be possible with the patient wearing a mask. For the Valsalva maneuver, the mouthpiece must be replaced between patients. A disposable viral filter should be used in between the mouthpiece and the pressure tubing. The increased airflow resistance introduced by an effective filter will not compromise the test result, since increased intrathoracic pressure, not exhaled pressure, is the stimulus for the hemodynamic response. The patient should be instructed to position the mouthpiece themselves and to hold it in place during maneuver, and ideally, staff should maintain a 2-m distance from the patient as they forcefully exhale.

If filters cannot be used, then the tubing will need to be replaced between patients, or cleaned if it is not disposable. If cleaning tubing or other parts, a cleaning solution with antiviral properties should be used that follows vendors' specifications for cleaning procedures and solutions. Cleaned parts should be dried before reconnection to avoid the risk of damaging the sensor in the data acquisition system. Cleansed parts can be air-dried, if a source of flowing air (not breath-blowing) is available, to help with drying. 


\section{Personal protective equipment (PPE)}

It is recommended that PPE be donned by all clinicians and healthcare providers during autonomic testing. This should include a face mask covering the nose and mouth (a procedural or surgical mask, not a cloth mask), protective eyewear (eye shields or face shield, not just eyeglasses), and disposable gloves and/or very frequent handwashing. All those in contact with patients should avoid touching their own faces during testing. No food or drink should be brought into the testing laboratory. Clinicians' hands should be washed before and after gloves are removed. In developing PPE procedures, consultation with the institution's infection control team regarding site-specific recommendations, including the need for additional PPE and workspace safety, is strongly recommended. Appropriate donning and doffing procedures should be followed as outlined by national and international public health agencies [7].

\section{Environmental cleaning}

Institutional recommendations for environmental cleaning and disinfection of the testing room should be followed. This will likely require more frequent and enhanced cleaning than was the norm prior to COVID-19. This should include all shared areas (waiting rooms, shared bathrooms).

\section{Know your local rules}

This guidance includes suggestions to help with the safe practice of autonomic testing. This document is not intended to be a substitute for professional medical judgement, advice, diagnosis, or treatment. Institutional, state, and federal policies should take precedent over this guidance document.

Author contributions JJF - None. WPC - None. VEC - Research grants from the Heart and Stroke Foundation of Canada, Craig $\mathrm{H}$
Nielsen Foundation, and International Collaboration On Repair Discoveries. LNK - None. AP - None. WS - None. HS - None. SV - SV has received research support from Dysautonomia International, Genentech, Grifols, Rex Griswold Foundation, and Athena/Quest Diagnostics and personal compensation for consulting for Argenx, Alexion, and Lundbeck. SV has provided medicolegal consultation related to HPV vaccination. SRR - SRR has grant support from the Canadian Institutes of Health Research (Ottawa, Canada), Cardiac Arrhythmia Network of Canada (London, ON, Canada), and Dysautonomia International (East Moriches, NY, USA). SRR is a consultant for Lundbeck LLC and Theravance Biopharma, and serves as DSMB Chair for a phase 2 study run by Arena Pharmaceuticals. SRR has received compensation for editorial activities (Associate Editor) with Autonomic Neuroscience: Basic and Clinical.

Funding This work was supported in part by the National Institutes of Health grant UL1 TR000445, and by the Canadian Institutes of Health Research (CIHR; Ottawa, Canada) grant MOP142426.

\section{References}

1. Centers for Disease Control and Prevention. Coronavirus disease 2019 (COVID-19): cases in the U.S. https://www.cdc.gov/coron avirus/2019-ncov/cases-updates/cases-in-us.html. Accessed date 30 Mar 2020.

2. World Health Organization. Coronavirus disease (COVID-19) situation reports. https://www.who.int/emergencies/diseases/novel -coronavirus-2019/situation-reports/. Accessed 8 June 2020.

3. Bai Y, Yao L, Wei T et al (2020) Presumed asymptomatic carrier transmission of COVID-19. JAMA 10:1. https://doi.org/10.1001/ jama.2020.2565.

4. Zou L, Ruan F, Huang M et al (2020) SARS-CoV-2 viral load in upper respiratory specimens of infected patients. New Eng J Med 382:1177-1179. https://doi.org/10.1056/NEJMc2001737.

5. van Doremalen N, Bushmaker T, Morris DH et al (2020) Aerosol and surface stability of SARS-CoV-2 as compared with SARSCoV-1. N Engl J Med 1:17. https://doi.org/10.1056/NEJMc20049 73

6. Low PA, Tomalia VA, Park KJ (2013) Autonomic function tests: some clinical applications. J Clin Neurol 9(1):1-8. https://doi. org/10.3988/jen.2013.9.1.1

7. Centers for Disease Control and Prevention. Coronavirus disease 2019 (COVID-19): using personal protective equipment (PPE) (https://www.cdc.gov/coronavirus/2019-ncov/hcp/using-ppe.html. 